\title{
Developing a Collaborative Study Protocol for Combining Payer-Specific Data and Clinical Trials for CER
}

\author{
Robert J. Sanchez, PhD; Jack Mardekian, PhD; Mark J. Cziraky, PharmD; and C. Daniel Mullins, PhD
}

$\mathrm{T}$ he demand for comparative effectiveness research (CER) by health care providers and payers represents new opportunities for the U.S. government, research organizations, and pharmaceutical companies to generate "meaningful evidence" for use in medical decision making. ${ }^{1}$ CER studies conducted with a payer perspective should develop questions, select outcomes, and utilize data that are applicable to the payers themselves for use with their formulary and reimbursement decision-making processes. CER studies for prescribers should be designed and implemented to inform evidence-based therapeutic guidelines, providing actionable information from their everyday practice use. The challenge is how to conduct CER studies that satisfy the simultaneous requirements of scientific rigor and applicability to the respective decision makers. One solution is to address the demand for "real-world" data (RWD) by involving decision makers and other key stakeholders early on in the development of the research designs and implementation of study protocols when conducting CER studies. RWD have been defined "as data used for decision-making that are not collected in conventional RCTs" (randomized controlled trials); ${ }^{2}$ therefore, the ability to gather input from the payer is essential to ensure collected endpoints are applicable to the decision makers themselves.

RCTs are considered the "gold standard" for providing evidence about a product's efficacy and are the basis for supporting formulary decision making. While the internal validity of RCTs is well known and established, the controlled protocols of RCTs may not have the desired level of external validity for a managed care organization's (MCO) population. Consequently, health care decision makers are examining other sources of data to supplement RCTs for their health care coverage policies. Health care providers and payers use available evidence from both RCTs and RWD sources to decide whether a particular drug product offers tangible clinical benefits and value compared with existing therapies. Improving medical outcomes and providing positive impact on health care expenditures are shared goals of providers, payers, and the pharmaceutical industry. ${ }^{3}$

\section{Developing CER Studies to Inform Payer Decision Making}

Payers are interested in CER results and evidence-based value assessments of comparator therapies to use in their coverage decision-making processes. Some have proposed that CER involving systematic reviews of effectiveness evidence could improve the coverage and reimbursement processes. ${ }^{4}$ However, now more than ever, there is a need for better evidence generation rather than just better synthesis of existing evidence, which raises the question of how more meaningful evidence could be generated and how the decision makers could be involved in the identification of evidence gaps, design of study protocols, and implementation of CER studies, particularly those that propose to use RWD. It also is important to determine when additional studies, and related designs, are needed; value of information analysis, which examines the value of generating new evidence for decision making, ${ }^{5}$ can assist in that process since there is a need to prioritize in addition to grading the quality of the evidence. ${ }^{6}$

Stakeholder engagement in CER is encouraged by the Agency for Healthcare Research and Quality (AHRQ). The selection of stakeholders and processes for engagement will continue to evolve. Stakeholder engagement will no doubt involve patients and physicians, yet when it comes to coverage and formulary decisions, it is clear that payers and other health care stakeholders have an interest in participating in the research design and conduct. In fact, a recent article that reports on key informant interviews from major U.S. payers documents their willingness to be involved in studies that address the value of drug therapies. ${ }^{7}$

\section{A Case Study in Neuropathic Pain}

The remainder of this paper describes a collaborative effort between a payer, a research organization (HealthCore), and a drug manufacturer-sponsor (Pfizer) to develop a study protocol that combines elements of an RCT with RWD sources to answer mutually aligned research questions. These types of collaborative research studies can never replace clinical trials done for regulatory approval and labeling; however, in the post-regulatory environment, they may provide supplemental evidence that is valued by some payers. The example of the collaborative development of a study protocol highlighted in this paper is from an ongoing study. Pfizer is currently working with a large MCO and a research organization, HealthCore, to examine the relationship of its medication utilization strategy for pregabalin to utilization and expenditures. Medication utilization strategies, such as prior authorization (PA) and step therapy, are effective tools used by payers to control medication costs or to control access to medications in which the potential for harm may outweigh the benefits. With respect to the former, studies of the impact of PA and step therapy on medical and/or total cost of care (pharmacy and medical cost) have shown mixed results with respect to overall savings. ${ }^{8-17}$

Recently, 2 Pfizer-sponsored retrospective studies examining the association of a pregabalin PA on the total cost of care in a Medicaid and a commercial population were presented to the MCO. ${ }^{16-17}$ Because the MCO did not believe that the studied population was representative of its beneficiaries, Pfizer and 
the MCO agreed to undertake a prospective study to answer the question of whether the PA on pregabalin would affect costs; the study uses the plan's beneficiaries and the physicians who treat the MCO's patients with painful diabetic peripheral neuropathy (pDPN) or fibromyalgia (FM).

The MCO's PA for pregabalin is paper-based and requires the physician to fax the PA form to the MCO. The specific requirements for a pregabalin approval include (a) certification of a diagnosis of FM, pDPN, postherpetic neuralgia (PHN), or epilepsy; (b) confirmation of pharmacy benefit eligibility and; (c) for patients with these diagnoses other than epilepsy, a trial of at least 180 days on a formulary agent approved for treating pain (e.g., tricyclic antidepressants, cyclobenzaprine, fluoxetine, trazodone). Pfizer, HealthCore, and the MCO agreed to study the effect of PA under "real world" conditions, using a hybrid between an RCT and an observational study, with randomization at the physician level. All parties also mutually agreed on endpoints consisting of health care costs and patientreported outcomes (PROs).

Process for Developing the Study Protocol. Before an appropriate study design was identified, a process was mutually developed to ensure that Pfizer and the MCO had equal decision-making authority and contribution into the research design with the research organization serving as operational hub of the project. A core study team of 10 researchers; 2 from the MCO, 3 from Pfizer, 4 from HealthCore, and 1 independent statistician was formed. Because the proposed study would most likely use a nontraditional study design, a scientific advisory board composed of 5 members, including 1 external methodologist and 2 clinical experts, as well as 1 contributor each from the MCO (medical director), and Pfizer (senior health economist) was established to help guide and advise the study design. In order to ensure parity in decision making, all organizations contributed to and agreed to the selection of the scientific advisory board members. A study outline was prepared once there was agreement on the framework for the study in order to obtain internal agreement within each organization to proceed with the study and to obtain necessary funding within Pfizer for the research conduct. The study protocol was written and endorsed by all participating collaborators. It is known as the ExPAND (Examination of Pregabalin Access for Treatment of Indicated Pain Disorders) study and is posted on www.clinicaltrials.gov as NCT01280747. Results will also be posted once the study data are analyzed according to the Statistical Analysis Plan. The stated hypothesis of study NCT01280747 is "that fibromyalgia (FM) and painful diabetic peripheral neuropathy (pDPN) patients with access restrictions on pregabalin will lead to higher healthcare resource use and cost compared to patients without such restrictions on pregabalin..."

Study Design. Much like the prior retrospective claims database studies, the objective of this study was to determine the impact of a PA on pregabalin, not a direct comparison of treatment effects of specific medications. It was clear to the research team that a study design was needed that would be feasible and test the impact of a PA on pregabalin. While a traditional RCT was preferred, this study design seemed unlikely since blinding and randomization to a group were not feasible. We also considered a pragmatic clinical trial (PCT), a type of RWD which aims at exploring a hypothesis and study design to inform decision making. ${ }^{2,18}$ While a PCT study design seemed most appropriate, the team wanted to go beyond the traditional definition of a PCT, which generally does not include aspects of retrospective data collection. Therefore, the collaborative research team proposed an observational PCT and also brought in retrospective data elements into the study (e.g., administrative claims for visits and charges) to better inform the payer in an economic decision. The retrospective component of the study was necessary to assess disease-related health care utilization and cost as well as total all-cause cost of care. The study design included a cluster randomization at the physician level in an attempt to reduce confounding, and endpoints were to be evaluated mainly through observational follow-up. The final study design was agreed upon by study team members at the MCO, HealthCore, and Pfizer and endorsed by the scientific advisory board. The study will enroll 2,280 patients from 228 physicians (i.e., 10 patients per physician) across the 14 states where the health plans have membership. The physicians will be randomized on a 1:1 basis to usual care (PA policies in place) or expanded access (non-PA group). Although all patients for the 114 physicians in the non-PA group can receive pregabalin without restriction (i.e., regardless of prior use of formulary medication and regardless of diagnosis), the 10 patients selected for each physician will be required to have a diagnosis of either FM or pDPN.

Physician and Patient Recruitment and Randomization. The retrospective elements in this study are utilized to inform aspects of the study including the primary endpoint, cost to treat FM and pDPN, and the identification of physicians treating FM or pDPN patients. Participating physicians are randomized to 1 of the 2 study arms, usual care or expanded access. The usual care group will continue to have a PA on pregabalin while the expanded access group will have no PA on pregabalin. Following the design of a PCT, the inclusion criteria were established to increase external validity. Therefore, all patients aged 18 years or older with a diagnosis of either FM or pDPN are considered eligible for the study if they (a) are newly prescribed treatment for their either FM or pDPN or (b) a change in existing treatment is needed due to lack of effectiveness on their current treatment as determined by the physician. Choice of treatment for either disease state is at the discretion of the physician and patient. Patients enrolling in the study are consented according to the approved institutional review board 
(IRB) protocol and followed for 6 months; however, following the pragmatic study design, patients will see the physician under routine care, and patient visits are not mandated beyond the baseline visit except for the end-of-study visit. Additionally, patients are not compensated for office visit care, nor are they compensated for the cost of prescription medications.

All patients in both the PA and non-PA groups will meet the PA criterion of a diagnosis of either FM or pDPN. The difference between the groups is that physicians in the non-PA group will be able to prescribe pregabalin without restrictions, if deemed appropriate, whereas physicians in the PA group will be required to (a) complete and fax the PA approval form and (b) document a trial of 180 days on a formulary agent (e.g., tricyclic antidepressants, cyclobenzaprine, fluoxetine, trazodone), to obtain coverage for a pregabalin should the physician prescribe pregabalin.

Measured Outcomes and Reporting of Assessment. All patients will be evaluated on 2 primary endpoints: pain-related patient-reported outcomes (numeric rating scale [NRS]) and all-cause health care resource costs (from administrative claims records). There are also a number of secondary outcomes measured including the Brief Pain Inventory, Fibromyalgia Impact Questionnaire (FM patients only), Work Productivity and Activity Impairment Questionnaire, and the Patient Global Impression of Change. ${ }^{19-22}$ Patients complete the instruments at baseline, month 1 , month 3 , and month 6 . However, as mentioned above, patients are not required to have office visits at the above time periods. As a result, subjects are given a binder with all the PRO instruments and will be instructed to mail (return postage provided) the PRO instruments directly to HealthCore. Alternatively, if patients have a scheduled visit within a 2-week time period of the schedule above, they will be asked to bring the instruments with them to the visit.

Database Development. All prospectively generated study data will be collected using electronic records (eCRFs) and will reside in a Health Insurance Portability and Accountability Act (HIPAA)-compliant secure database. A data management plan will be developed with cleaning and validation instructions consistent with both traditional clinical trial and real world data.

Study Limitations. All CER studies have limitations and potential biases. The current study was designed to limit these biases while attempting to balance internal and external validity; nonetheless, biases remain, and publication and dissemination of the study results will need to address these biases. The non-PA group will have the entire restriction lifted while the PA group will continue to have the PA in place for pregabalin. While patients in this study may meet the MCO's criteria for pregabalin, it is hypothesized that many physicians in the PA group will not prescribe pregabalin due to the process of getting the medication. Furthermore, the fact that the study focuses only on patients with FM or pDPN reduces the ability to fully assess the potential cost implications of a PA program on pregabalin since the drug may be prescribed for patients who do not meet the labeled indications.

Benefits to Participating Organizations. Manufacturers and payers have a mutual interest in conducting CER studies that inform coverage and reimbursement decisions. The current study provides benefits to both Pfizer and the participating MCO. As a participating partner, the MCO benefits through its ability to conduct a CER study on its own enrollee population with financial support from Pfizer. Historically, many pharmacoeconomic studies were designed by the sponsoring manufacturer, and the majority of "input" from the MCO was the use of its administrative claims. In contrast, the current study integrates the MCO as an equal partner in the study design and conduct. Furthermore, there is a prospective data capture component to expand outcomes to include patientcentered outcomes using validated instruments. As a sponsor, Pfizer benefits from the assurance that the study will produce "meaningful" evidence since the MCO participated in the design and execution of the study, as well as demonstrate its leadership in collaborative CER design and conduct. Another benefit is the insight the pharmaceutical sponsor gains on the MCO decision-making process regarding a payer's requirements to establish PA, step-therapy edits, and other utilization control tools that are used routinely by MCOs. Finally, from an "internal management" perspective, CER researchers at Pfizer were able to provide exposure to their clinical trial specialist colleagues at Pfizer, whose focus is primarily on regulatory approval, to key post-approval research requirements which are being requested by many payers. Thus, the clinical trials group at Pfizer obtains first-hand knowledge of the potential benefits of RWD sources to assess effectiveness.

\section{Conclusion and Next Steps}

The increasing demand for CER studies and evidence of comparative clinical benefits and value likely will be addressed through continued development of novel approaches to CER studies that involve decision maker participation. Moving forward, CER protocols that are jointly designed and conducted by manufacturers and payers likely will attempt to combine the best concepts from clinical trials and analysis of RWD. This effort will require scientifically rigorous investigations that produce meaningful evidence in an efficient manner. The results will supplement prior evidence from RCTs and provide additional information for payers to potentially aid in coverage determination. There no doubt will be a variety of case studies, such as the one described in this article. These early CER endeavors will provide insights for enhancing CER methods and the entire evidence generation process. The pDPN and FM study described in this article is expected to be completed in 
mid-2012. The study team along with the scientific advisory board will work to determine an appropriate venue to disseminate the results, which will shed light not only on the specific research being addressed but also on the approach to conducting collaborative CER studies.

\section{Authors}

ROBERT J. SANCHEZ, PhD, is Director, U.S. Health Economics and Outcomes Research, and JACK MARDEKIAN, PhD, is Senior Director, OR Statistical Scientist, Pfizer Inc, New York, New York. MARK J. CZIRAKY, PharmD, is Vice President, HealthCore, Inc. Wilmington, Delaware. C. DANIEL MULLINS, PhD, is Professor, Pharmaceutical Health Services Research Department, University of Maryland School of Pharmacy, Baltimore, Maryland.

\section{DISCLOSURES}

This supplement was funded by Pfizer. Sanchez and Mardekian are Pfizer employees. Cziraky reported that HealthCore receives funding for research projects from pharmaceutical companies including Pfizer. Mullins reported financial and other relationships with Pfizer that include receipt of grants, consulting fees or honoraria, support for travel, consulting fees for participation in review activities such as data monitoring boards, payment for writing or reviewing the manuscript, advisory board membership, payment for lectures including service on speakers bureaus, and payment for development of educational presentations.

The 4 authors contributed equally to writing and revision of the manuscript.

\section{ACKNOWLEDGEMENTS}

The authors would like to acknowledge the helpful comments from Shelly Stanley, MS, RD; Richard J. Willke, PhD; and Zhanna Jumadilova, MD, MBA.

\section{REFERENCES}

1. Owens DK, Qaseem A, Chou R, Shekelle P; Clinical Guidelines Committee of the American College of Physicians. High-value, cost-conscious health care: concepts for clinicians to evaluate the benefits, harms, and costs of medical interventions. Ann Intern Med. 2011;154(3):174-80.

2. Garrison LP Jr, Neumann PJ, Erickson P, Marshall D, Mullins CD. Using real-world data for coverage and payment decisions: the ISPOR RealWorld Data Task Force report. Value Health. 2007;10(5):326-35. Available at: http://download.journals.elsevierhealth.com/pdfs/journals/1098-3015/ PIIS1098301510604706.pdf. Accessed September 21, 2011.

3. Nayer C. The value of dividends in health: a call to align stakeholders. Clin Ther. 2009;31(11):2689-96.

4. Pearson SD, Bach PB. How Medicare could use comparative effectiveness research in deciding on new coverage and reimbursement. Health Aff (Millwood). 2010;29(10):1796-804.
5. Groot Koerkamp B, Myriam Hunink MG, Stijnen T, Weinstein MC. Identifying key parameters in cost-effectiveness analysis using value of information: a comparison of methods. Health Econ. 2006;15(4):383-92. 6. Meltzer D, Basu A, Conti R. The economics of comparative effectiveness studies: societal and private perspectives and their implications for prioritizing public investments in comparative effectiveness research. Pharmacoeconomics. 2010:28(10):843-53.

7. Mullins CD, Ratner J, Ball DE. How do U.S. payers react to and use pharmacoeconomic information? International Journal of the Economics of Business. 2011; (in press)

8. Motheral BR, Henderson R, Cox ER. Plan-sponsor savings and member experience with point-of-service prescription step therapy. Am J Manag Care. 2004;10(7 Pt 1):457-64

9. Yokoyama K, Yang W, Preblick R, Frech-Tamas F. Effects of a step-therapy program for angiotensin receptor blockers on antihypertensive medication utilization patterns and cost of drug therapy. J Manag Care Pharm. 2007;13(3):235-44. Available at: http://www.amcp.org/data/jmcp/235-44. pdf.

10. Dunn JD, Cannon E, Mitchell MP, Curtiss FR. Utilization and drug cost outcomes of a step-therapy edit for generic antidepressants in an HMO in an integrated health system. J Manag Care Pharm. 2006;12(4):294-302. Available at: http://amcp.org/data/jmcp/research_294-302.pdf.

11. Hartung DM, Touchette DR, Ketchum KL, Haxby DG, Goldberg BW. Effects of a prior-authorization policy for celecoxib on medical service and prescription drug use in a managed care Medicaid population. Clin Ther. 2004;26(9):1518-32.

12. Delate T, Mager DE, Sheth J, Motheral BR. Clinical and financial outcomes associated with a proton pump inhibitor prior-authorization program in a Medicaid population. Am J Manag Care. 2005;11(1):29-36.

13. Gleason PP, Williams C, Hrdy S, Hartwig SC, Lassen D. Medical and pharmacy expenditures after implementation of a cyclooxygenase-2 inhibitor prior authorization program. Pharmacotherapy. 2005;25(7):924-34.

14. Law MR, Lu CY, Soumerai SB, et al. Impact of two Medicaid priorauthorization policies on antihypertensive use and costs among Michigan and Indiana residents dually enrolled in Medicaid and Medicare: results of a longitudinal, population-based study. Clin Ther. 2010;32(4):729-41.

15. Louder AM, Joshi AV, Ball AT, et al. Impact of celecoxib restrictions in Medicare beneficiaries with arthritis. Am J Manag Care. 2011;17(7):503-12.

16. Margolis JM, Johnston SS, Chu BC, et al. Effects of a Medicaid prior authorization policy for pregabalin. Am J Manag Care. 2009;15(10):e95-102.

17. Margolis JM, Cao Z, Onukwugha E, et al. Healthcare utilization and cost effects of prior authorization for pregabalin in commercial health plans. Am J Manag Care. 2010;16(6):447-56.

18. Tunis SR, Stryer DB, Clancy CM. Practical clinical trials: increasing the value of clinical research for decision making in clinical and health policy. JAMA. 2003;290(12):1624-32.

19. Cleeland CS, Ryan KM. Pain assessment: global use of the Brief Pain Inventory. Ann Acad Med Singapore. 1994;23(2):129-38.

20. Reilly MC, Zbrozek AS, Dukes EM. The validity and reproducibility of a work productivity and activity impairment instrument. Pharmacoeconomics. 1993;4(5):353-65

21. Burckhardt CS, Clark SR, Bennett RM: The fibromyalgia impact questionnaire: development and validation. J Rheumatol. 1991;18(5):728-33.

22. The Patients' Global Impression of Change (PGIC) scale. Available at: http://www.chiroplushealthcare.com/PGIC.PDF. Accessed May 27, 2011. 